\title{
Norois
}

Environnement, aménagement, société

245 | 2017

Adapter les territoires aux changements climatiques : transition urbanistique et aménagement de l'espace

\section{Note de recherche. Plaidoyer pour le développement d'une action climatique régionale : aménagement de l'espace, usage des sols et conditions atmosphériques}

Advocating for the development of a regional climate action: spatial planning, land-use and atmospheric conditions

Mathieu Perrin, Nathalie de Noblet-Ducoudré, Susanna Strada et André Torre

\section{(2) OpenEdition}

\section{Édition électronique}

URL : https://journals.openedition.org/norois/6218

DOI : $10.4000 /$ norois. 6218

ISBN : 78-2-7535-7465-6

ISSN : $1760-8546$

\section{Éditeur}

Presses universitaires de Rennes

Édition imprimée

Date de publication : 31 décembre 2017

Pagination : $29-45$

ISBN : 978-2-7535-7456-4

ISSN : 0029-182X

\section{Référence électronique}

Mathieu Perrin, Nathalie de Noblet-Ducoudré, Susanna Strada et André Torre, « Note de recherche. Plaidoyer pour le développement d'une action climatique régionale : aménagement de l'espace, usage des sols et conditions atmosphériques », Norois [En ligne], 245 | 2017, mis en ligne le 31 décembre 2019, consulté le 13 janvier 2022. URL : http://journals.openedition.org/norois/6218 ; DOI : https:// doi.org/10.4000/norois. 6218 


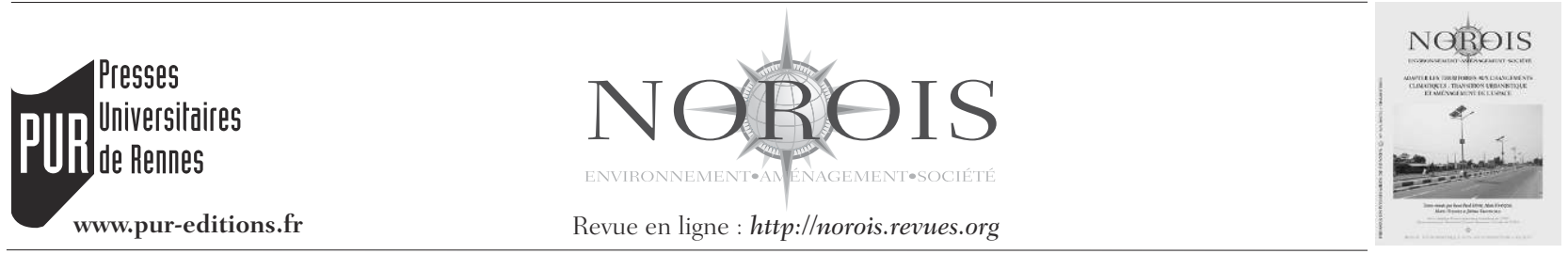

\title{
Note de recherche \\ Plaidoyer pour le développement d'une action climatique régionale : aménagement de l'espace, usage des sols et conditions atmosphériques
}

\author{
Research note - Advocating for the Development of a Regional Climate Action: \\ Spatial Planning, Land-use and Atmospheric Conditions
}

\author{
Mathieu Perrin ${ }^{a}$, Nathalie de Noblet-Ducoudré ${ }^{\mathrm{b}}$, \\ Susanna STRADA ${ }^{\mathrm{c}}$ et André TorRe ${ }^{\mathrm{d}}$
}

\footnotetext{
a Auteur correspondant : Tel : +33 (0) 149596961 , LabEx BASC, UMR SADAPT, INRA, AgroParisTech, université Paris-Saclay, Paris, France; Chercheur associé à l'UMR Pacte. (mathieu.perrin@versaille.inra.fr)

${ }^{b}$ Laboratoire des Sciences du Climat et de l'Environnement, co-coordinatrice du LabEx BASC, UMR CEA-CNRSUVSQ, université Paris Saclay, 91191 Gif-sur-YvetTe cedex, France.

' LabEx BASC, Laboratoire des Sciences du Climat et de l’Environnement, UMR CEA-CNRS-UVSQ, université Paris Saclay, 91191 Gif-Sun-YvetTe cedex, France.

${ }^{\mathrm{d}}$ UMR SADAPT, INRA, AgroParisTech, université Paris-Saclay, Paris, France.
}

Résumé : Suite à une prise de conscience quant à la problématique du réchauffement global et à la reconnaissance d'une responsabilité anthropique dans le phénomène, la question climatique s'est progressivement inscrite au cœur des préoccupations aménagistes. Une attention toute particulière a notamment été portée aux dispositions spatiales et urbaines susceptibles de réduire les émissions de gaz à effet de serre, la démarche se voulant alors une contribution à l'effort mondial en matière d'atténuation du changement climatique. En outre, dans un souci d'adaptation, les aménageurs montrent peu à peu un regain d'intérêt pour des principes de climatologie urbaine et de bioclimatologie dans des agglomérations qui pourraient bien voir leurs conditions atmosphériques se réchauffer. Il en va du confort et de la santé de nombreuses populations. À travers cette note, nous soutenons, notamment à destination d'un public d'aménageurs, la possibilité d'étendre le champ de l'action climatique en développant une capacité à influencer les conditions atmosphériques à une échelle qualifiable de régionale ou d’intermédiaire. Différentes recherches en climatologie régionale ont en effet pu mettre en exergue l'influence significative que peuvent avoir les changements d'usage et de gestions des sols sur la météorologie et la qualité de l'air d'une région en raison des échanges - énergétiques, hydriques et chimiques - qui s'opèrent entre la surface et l'atmosphère. Nous exposons, sur la base de cas d'étude sélectionnés dans la littérature académique, quelles pourraient être les modalités d'une telle action climatique régionale, puis pointons quelques-uns des défis à relever pour que celle-ci puisse voir le jour.

Abstract: As a result of the growing awareness regarding the global warming problem and its human causes at the end of the $20^{\text {th }}$ century, climate issue has progressively become a prominent focus of spatial planning. A particular attention has been paid to spatial configurations and urban designs that are liable to curb greenhouse gas emissions, with the intent of contributing to the global effort in terms of climate change mitigation. Moreover, spatial planners show a renewed interest in principles of urban climatology in cities and metropolitan areas over which atmospheric conditions are likely to become hotter in the future decades. Therefore, the comfort of living and the health of many people are at stake. This note supports the possibility of extending the scope of climate action by developing a new ability in influencing atmospheric conditions at a regional/intermediary scale. Various researches in regional climatology have highlighted the 
substantial influence land uses and land cover can have on the weather and the air quality of a region due to the - energetic, hydric and chemical - interactions between the surface and the atmosphere. On the basis of case-studies selected in the scientific literature, this note presents what could be the main features of such a climate action at the regional scale and then highlights some challenges to be faced to make it operational.

Mots clés : changement climatique - climatologie - qualite de l'air - îlot de chaleur - occupation du sol - impacts environnementaux et socio-economiques

Keywords: climate change, climatology - air quality - urban heat island - land use-environmental and socio-economical impacts

\section{INTRODUCTION}

À la fin du siècle dernier, et notamment au travers du Protocole de Kyoto signé en 1997, s’est opérée une prise de conscience quant à la problématique du réchauffement global. La question climatique a alors gagné en importance dans les préoccupations aménagistes et planificatrices, (Bulkeley, 2006; Wilson et Piper, 2006; Davoudi et al., 2009) et cela à deux titres. Premièrement, dans une logique d'atténuation du changement climatique et donc de contribution à un effort global, une attention croissante a été portée aux solutions permettant de réduire les émissions de gaz à effet de serre. En ce sens, des efforts ont pu être menés en matière d'énergétique, que ce soit de manière plus directe avec par exemple l'inscription dans les tissus urbains de smart grids, réseaux de chaleur et autres solutions permettant d'optimiser la production, la distribution, le stockage et la consommation énergétiques - ou plus indirecte - à travers des actions visant à améliorer l'isolation et l'inscription bioclimatique des bâtiments ou encore des développements sur les formes urbaines et mobilités attachées à celles-ci. Deuxièmement, dans une logique d'adaptation, la réflexion s'est ensuite tournée vers des configurations spatiales et urbaines susceptibles de réduire, à une échelle essentiellement locale, la vulnérabilité des territoires et des écosystèmes aux effets attendus de ce changement climatique. Les aménageurs portent par exemple leur attention sur la question des risques, redoutés sur la base d'une probable multiplication et intensification des aléas météorologiques ou encore de possibles submersions des terres en raison d'une élévation du niveau des océans. Si la plupart des mesures d'adaptation proposées s'avèrent " passives ", dans le sens où elles n'ont pas pour objectif d'agir sur le climat lui-même, certaines s'inscrivent dans le registre de l'action cli- matique à proprement parler. C'est tout particulièrement le cas des orientations prises en matière d'aménagement et de planification pour modérer l'effet d'îlot de chaleur urbain (ICU) ${ }^{1}$, cela dans l'idée d'assurer entre autres la santé et le confort de vie des populations.

Dans cette note, nous discutons la possibilité d'étendre le champ de l'action climatique en considérant des échelles spatiales et par conséquent des leviers jusqu'alors non ou peu mobilisés dans la pratique aménagiste. Les développements en matière de climatologie locale à régionale ont mis en exergue l'influence significative que peuvent avoir l'occupation des sols ainsi que leurs modalités de gestion sur la météorologie, le climat ${ }^{2}$ ou encore la qualité de l'air - cela sur des distances allant du kilomètre à plusieurs dizaines ou même centaines de kilomètres ${ }^{3}$ - en raison des échanges énergétiques, hydriques et chimiques qui s'opèrent entre la surface et l'atmosphère (Raddatz, 2007; Pielke et al., 2011 ; Pitman et de Noblet-Ducoudré, 2011 ; Mahmood et al., 2014; Heald et Spracklen, 2015). Ces différents travaux permettent d'une part de prendre conscience de l'impact, souvent mésestimé, que peuvent avoir les sociétés humaines sur leurs conditions de vie (températures, précipitations, vents, etc.) en occupant l'espace et en usant des sols pour différentes finalités fonctionnelles (résidentiel, agricole, etc.). Le

1. Lî̀lot de chaleur urbain désigne une étendue, sous influence de la ville, sur laquelle les températures observées - au niveau du sol, de la canopée ou encore de la couche limite - s'avèrent plus chaudes que sur les superficies rurales alentours

2. Météorologie et climatologie se distinguent essentiellement par leurs échelles de temps, la première s'attachant à l'évolution du temps aux échelles diurnes, saisonnières et interannuelles. La seconde s'intéresse à l'état moyen des variables atmosphériques sur des pas de temps d'une à plusieurs décennies. Les normales climatologiques sont traditionnellement définies sur une période de trente ans. Une modification durable de la météorologie par des actions aménagistes aura un effet à long terme et donc sur le climat.

3. À ces échelles, les écoulements atmosphériques sont particulièrement sensibles aux spécificités topographiques et à l'agencement des surfaces (villes, prairies, cultures, forêts, etc.). 
développement de ces connaissances scientifiques en matière d'interactions surface-atmosphère laisse entrevoir de possibles traductions opérationnelles.

L'extension du domaine de l'action climatique que nous proposons intégrerait une capacité à influencer les conditions atmosphériques non pas aux échelles urbaine ou globale, plus fréquemment considérées par les aménageurs, mais à une échelle qualifiable de régionale ou d'intermédiaire. Elle prendrait la forme d'une démarche de planification se souciant de l'usage des sols, de leurs couverts végétaux ainsi que de leurs conséquences atmosphériques sur des périmètres élargis - de plusieurs centaines à plusieurs milliers de $\mathrm{km}^{2}$ - comprenant espaces urbains, périurbains et ruraux. Cette nouvelle échelle permettrait notamment de considérer dans l'action climatique des superficies et par conséquent des couverts végétaux, qu'ils soient agricoles ou naturels, généralement peu pris en compte dans la pratique ou sinon dans l'idée qu'ils puissent stocker du carbone dans le cadre de la lutte contre l'effet de serre et donc contre le réchauffement global. Par conséquent, ce sont également de nouveaux leviers en matière d'action climatique, spécifiés ultérieurement pour certains d'entre eux, qui seraient à considérer. Quelques-uns de ces leviers ont déjà pu être identifiés et investigués par les chercheurs spécialisés sur les interactions surface-atmosphère. En revanche, la transposition opérationnelle de ces développements scientifiques n’a pas encore eu lieu.

Les pages qui suivent visent à présenter, à l'attention tout particulièrement d'un public d'aménageurs, les fondements et modalités de cette possible action climatique d'échelle intermédiaire. À dessein, il sera tout d'abord fait état des démarches menées au niveau de villes ou même plus récemment de régions urbaines afin de montrer comment ont été appréhendées jusqu'alors dans la pratique les interactions surfaces-atmosphère et par conséquent l'impact des orientations aménagistes sur l'environnement proche (section 1). Puis, seront exposés certains des fondements scientifiques, notamment sur la base de cas d'étude extraits de la littérature académique en matière de climatologie régionale, laissant envisager de nouvelles opportunités pour influer sur le climat, la météorologie et la qualité de l'air de par une plus grande attention portée à l'usage des sols ainsi qu'à leurs modalités de gestion (section 2). Enfin, seront spécifiées quelques-unes des conditions nécessaires et défis à relever pour que puisse voir le jour cette extension du domaine de l'action climatique à une échelle régionale (section 3).

\section{UN INTÉRÊT PRÉEXISTANT POUR LES PROBLÉMATIQUES CLIMATIQUES EN MILIEUX URBAINS}

L'intérêt pour les impacts atmosphériques imputables à l'urbanisation n'est pas nouveau, de nombreuses mesures de températures, précipitations, conditions venteuses ayant notamment été réalisées - et parfois comparées à des mesures effectuées en périphérie - en diverses agglomérations européennes au cours du XIX ${ }^{\mathrm{e}}$ siècle (Hebbert et Jankovic, 2013). Il faut toutefois attendre le vingtième siècle pour voir se structurer un réel champ scientifique en la matière, ainsi que des mesures plus systématique et des outils opérationnels se diffusant auprès des aménageurs et planificateurs.

\section{Développement et diffusion des principes de climatologie urbaine}

En 1938, la municipalité de Stuttgart - concernée par d'importants problèmes de pollution de l'air en raison notamment d'une localisation en fond de vallée encaissée limitant les circulations d'air et la vitesse des vents - engage dans son agence de protection environnementale un météorologue ayant à étudier les impacts du plan d'urbanisme local édité trois ans plus tôt. Son travail conduit à l'identification de couloirs canalisant l'air froid et donc susceptibles de venir rafraîchir et renouveler l'air pollué présent sur l'agglomération, qui reçoivent une attention toute particulière dans le plan d'urbanisme général adopté pour la ville en 1948 (Hebbert et Webb, 2012).

Au milieu du $\mathrm{xx}^{\mathrm{e}}$ siècle, des agglomérations telles que Los Angeles (1943), Londres (1952, 1955) ou New York $(1966,1970)$ se voient confrontées à d'intenses épisodes de smogs (Bach, 1972) qui amènent le grand public à prendre conscience des enjeux en matière de qualité de l'air. Suite notamment à l'épisode de 1966, dû à un phénomène de couche d'inversion ${ }^{4}$ résultant de conditions météorologiques

\footnotetext{
4. Une couche d'inversion désigne une couche de l'atmosphère dans laquelle la température croît avec l'altitude, en contradiction avec la baisse thermique qui s'observe normalement dans la troposphère. Le gradient de température se voit ainsi inversé.
} 
spécifiques limitant le mélange vertical des masses d'air et donc la dispersion des polluants, la municipalité new yorkaise décide d'intégrer dans ses services un météorologue. C'est souvent en réponse à des situations alarmantes en matière de pollution de l'air, et par conséquent de santé publique, que les collectivités - essentiellement nord-américaines ou européennes - apportent plus d'attention aux éléments de météorologie et de climatologie urbaines (Hebbert et Jankovic, 2013). Toutefois, durant une bonne partie de la seconde moitié du siècle, ces considérations s'effacent assez largement des préoccupations politiques et planificatrices locales, alors même que se développent les connaissances scientifiques en la matière (Hebbert et Webb, 2012; Hebbert, 2014).

C'est au tournant du XXI ${ }^{\mathrm{e}}$ siècle, dans un contexte de réchauffement global, que les aspects de météorologie et de climatologie urbaines regagnent progressivement en intérêt chez les décideurs publics et dans les collectivités territoriales. Les connaissances acquises, depuis de longues décennies déjà, en matière d'îlots de chaleur urbains et d'aménagements susceptibles de limiter le phénomène trouvent davantage d'écho, alors que des approches plus appliquées voient le jour. Entre autres, la classification des tissus urbains par zones climatiques locales 5 (Stewart et Oke, 2012), offrant une première idée des impacts en fonction des occupations des sols et morphologies bâties, permet à des agglomérations d'appréhender plus aisément la problématique de l'îlot de chaleur urbain (Cordeau, 2014). S'inspirant d'une méthodologie développée à partir des années 1970 par le service de climatologie urbaine de la ville de Stuttgart, diverses agglomérations européennes (Essen, Dortmund, Bochum, Bâle, Graz, Munich, Berlin, Lisbonne, Arnhem), asiatiques (Osaka, Fukuoka, Kobe, Yokohama, Hong Kong) ou sud-américaines (Salvador) se lancent

5. Les zones climatiques locales se conçoivent comme des unités spatiales au sein desquelles la température de l'air, généralement enregistrée à 1,5 ou 2 mètres du sol, s'avère uniforme sous des conditions de ciel clair et calme. Elles se différencient les unes des autres sur la base de propriétés surfaciques - ayant trait à la couverture des sols, à la morphologie bâtie et végétale ou encore aux émissions de chaleur anthropiques - qui viennent influencer la température de l'air. Initialement développé par des chercheurs spécialistes du phénomène d'îlot de chaleur urbain, souhaitant fournir un cadre d'analyse offrant de dépasser les imprécisions relatives à la trop simpliste opposition urbain-rural prise traditionnellement pour référence, le système de classification par zones climatiques locales a ensuite été repris dans les champs de l'urbanisme ou encore de l'écologie du paysage. également dans l'élaboration de cartes climatiques urbaines visant à transmettre aux urbanistes une représentation simplifiée, en deux dimensions, de phénomènes et problématiques climatiques à prendre en compte dans les orientations d'aménagement (Ren et al., 2010; Ng et Ren, 2015).

\section{Aspirations à une action climatique d'échelle métropolitaine}

Plus récemment, il s'observe une tendance, assez timide encore, à vouloir agir sur le climat, la météorologie et la qualité de l'air des milieux urbains en étendant le périmètre géographique à considérer. Il a par exemple été suggéré de tirer profit d'une planification territoriale élargie, à l'échelle du Grand Paris, pour faire notamment face à lî̀lot de chaleur urbain sur le cœur d'agglomération et ainsi réduire sa vulnérabilité dans un contexte de réchauffement global (Masson et al., 2013). En d'autres termes, alors qu'avait jusqu'à présent été plus sérieusement considéré l'effet rafraîchissant de parcs de tailles réduites et disséminés à travers le tissu urbain, il est désormais proposé de porter une attention à ces vastes superficies naturelles/agricoles périphériques et à leur influence tout aussi marquée, voire davantage, sur les températures du cœur d'agglomération. Il a été établi qu'un parc d'environ 150 hectares pouvait impacter les températures sur environ un kilomètre de distance (Upmanis et al., 1998). De manière plus aboutie, la région métropolitaine stuttgartoise a publié, s'appuyant sur son expérience déjà ancienne en matière de climatologie et de flux d'air en milieu urbain, un atlas climatique à l'échelle de la région urbaine élargie reposant sur une approche par les climatopes $^{6}$ (figure 1 - planche III, Baumüller et al., 2008). La stratégie adoptée est assez similaire dans l'esprit à ce qui fut réalisé par le passé sur l'agglomération, à savoir lutter contre l'îlot de chaleur urbain et les pollutions de l'air sur les superficies bâties, en cherchant notamment à favoriser

\footnotetext{
6. Les climatopes se conçoivent comme des unités spatiales aux caractéristiques microclimatiques homogènes et offrant une première idée de l'influence que peuvent avoir les superficies, leurs activités et leurs couverts sur les conditions atmosphériques. L'approche permet, à but analytique, une classification de l'espace susceptible de varier selon les démarches, contextes et besoins, bien que furent établis des systèmes standardisés pour les besoins notamment de la planification. Les climatopes sont généralement déterminés en fonction d'aspects relatifs à l'usage des sols, parfois à la situation topographique et à l'exposition, et à des caractéristiques surfaciques influençant les flux avec l'atmosphère.
} 
l'écoulement de masses d'air frais depuis les superficies agricoles et naturelles, cela afin de rafraîchir, dépolluer et prévenir les couches d'inversion. Cette réflexion a par la suite donné lieu à des préconisations d'aménagement, indiquant entre autres les espaces jugés d'importance et devant être préservés en l'état ou encore les localisations et orientations de bâti à privilégier sur les futurs développements pour faciliter, ou du moins ne pas entraver, certains flux d'air jugés importants.

Le cas stuttgartois est intéressant en ce qu'il expose la pertinence à concevoir l'action sur le climat, la météorologie et la qualité de l'air des agglomérations tout en portant une attention marquée aux superficies alentours. L'approche proposée à travers notre contribution s'inscrit assez largement dans cette logique, mais en intégrant dans l'équation des aspects jusqu'alors négligés. D’une certaine manière, nous proposons de passer d'une climatologie urbaine étendue à une climatologie régionale. $\mathrm{La}$ formulation ne doit pas seulement se comprendre d'un point de vue géographique, traduisant alors un changement dans l'échelle spatiale considérée de même qu'une plus grande attention aux superficies naturelles, agricoles ou bâties présentes en milieux rural et périurbain. Mais elle doit aussi s'entendre du point de vue des leviers susceptibles d'être mobilisés dans le cadre d'une action planificatrice ou aménagiste.

\section{INFLUENCE DES SOLS ET DES ORIENTATIONS AMÉNAGISTES SUR LES CONDITIONS ATMOSPHÉRIQUES}

Les professionnels de l'aménagement ont été conduits à prendre progressivement conscience de l'impact que peuvent avoir leurs interventions sur le climat et la qualité de l'air en milieux urbains. Il demeure néanmoins un décalage relativement important entre les connaissances développées dans le monde académique en matière d'interactions surface-atmosphère et celles qui peuvent être mobilisées à but opérationnel. Ce sont notamment des échelles spatiales et leviers d'action, fréquemment appréhendés dans le contexte scientifique, qui ne sont pas encore ou incomplètement exploités sur le terrain. Cette partie vise à restituer sommairement quelques principes généraux en matière d'interactions surface-atmosphère, puis à exposer des cas d'étude extraits de la littérature académique et susceptibles d'intéresser l'action aménagiste.

\section{Interactions surface-atmosphère}

Toute surface en contact avec l'atmosphère est en interaction permanente avec celui-ci via des processus tels que ceux qui président au cycle de l'eau (l'évaporation) ou au cycle du carbone (la photosynthèse et la respiration). Un changement de surface provoque par conséquent une diversité d'impacts sur le sol, le couvert végétal et l'air ambiant : une déforestation pourra induire des effets réchauffant/refroidissant, humidifiant/asséchant, polluant/ dépolluant (figure 2). Le signe de chaque effet, tout comme son intensité, est fonction des conditions météorologiques (chaud/froid, avec/sans neige, avec/ sans pluie, vent ou non), de la nature de la surface (arbres, herbacées, bitume) et de son état ( $\mathrm{sec} /$ mouillé, feuillage abondant/clairsemé). L'interaction surface-atmosphère doit donc se concevoir comme dynamique, ce d'autant plus sur des milieux fortement végétalisés. Ces processus entre surface et atmosphère s'inscrivent d'une part dans le registre biophysique et d'autre part dans le registre biogéochimique.

(a) Les processus dits biophysiques réfèrent aux modalités selon lesquelles énergie et eau s'échangent entre - et sont stockées par - la surface et l'atmosphère. La surface reçoit de l'énergie sous forme de rayonnement et en perd, donc se refroidit, essentiellement sous deux formes : par rayonnement, en fonction de sa température, mais également par génération de flux convectifs. Ces derniers contribuent directement au réchauffement de l'air ambiant, à son humidification, et au réchauffement ou refroidissement de l'atmosphère bien au-delà de l'air ambiant lors de processus de condensation ou de formation nuageuse (Seneviratne et al., 2010). Les bilans d'énergie et d'eau d'une surface sont très étroitement corrélés via l'évapotranspiration.

(b) Les processus dits biogéochimiques réfèrent aux modalités selon lesquelles éléments et composés chimiques s'échangent entre, se voient transformés et sont stockés par la surface et l'atmosphère. Il en va notamment des processus qui président aux échanges de dioxyde de carbone $\left(\mathrm{CO}_{2}\right)$, à savoir la photosynthèse et la respiration des plantes et des sols. Ils contribuent aux fonctions puits et source 


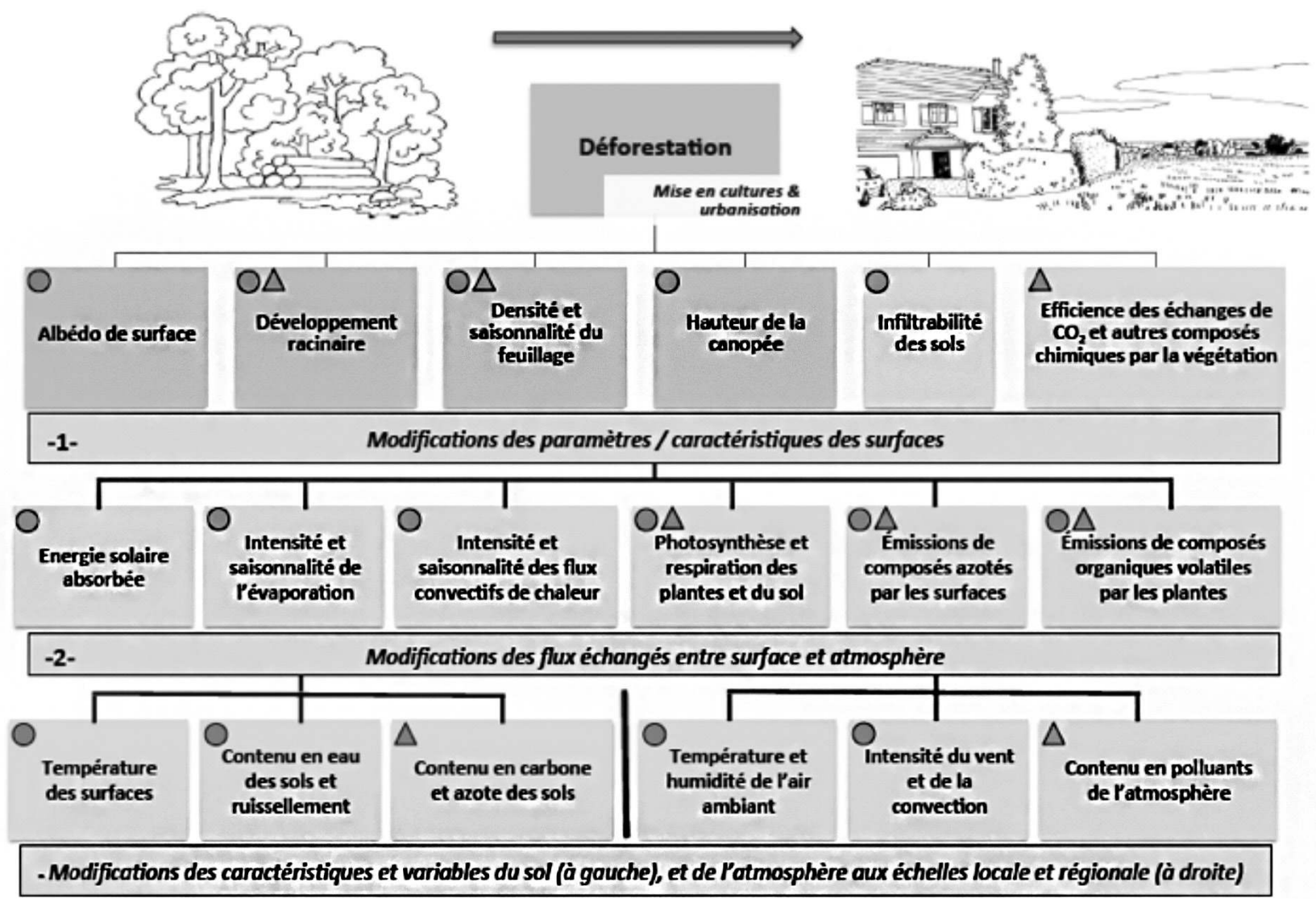

Figure 2 : Implications d'une déforestation sur les caractéristiques surfaciques (étape 1), les flux échangés entre la surface et l'atmosphère (étape 2) et sur les caractéristiques atmosphériques (étape 3)

Les cercles permettent d'identifier les variables/flux intervenant dans les processus biophysiques tandis que les triangles pointent sur les processus biogéochimiques.

Implications of a deforestation on the land-surface characteristics (step 1), the fluxes exchanged at the land-atmosphere interface (step 2) and on the atmospheric characteristics (step 3)

de carbone du système sol-plante, largement mis en avant au titre de la stratégie d'atténuation du changement climatique terrestre [http://www.un-redd. org/; http://4p1000.org/]. Cela concerne également des processus influençant fortement la qualité de l'air ambiant. Certains arbres ou arbustes émettent des quantités importantes de composés organiques volatiles dits d'origine biogénique (bio-COV) car produits naturellement par les plantes. Les prairies et champs cultivés en émettent également, bien que dans une moindre mesure. Ces composés participent, par l'intermédiaire de réactions physicochimiques, à la formation de particules atmosphériques considérées comme polluantes. Ils sont par ailleurs impliqués dans la formation d'ozone troposphérique, un gaz qui se révèle nocif pour l'homme et les plantes lorsque présent à proximité de la surface terrestre (Sillman, 1999). Si certaines espèces d'arbres et arbustes montrent ainsi, en raison de leurs émissions, un potentiel de dégradation de la qualité de l'air (Chameides et al., 1988; Corchnoy et al., 1992), d'autres surfaces arborées peuvent a contrario jouer un rôle dans sa dépollution. Les surfaces foliaires constituent en effet, en l'absence de précipitations, des zones de dépôt sec, entre autres pour les particules atmosphériques et l'ozone troposphérique (Nowak et al., 2000).

Cette figure 2 illustre à quel point les surfaces et notamment leurs couvertures végétales peuvent affecter la température, l'humidité ou encore la qualité de l'air. Ce sont non seulement les milieux ambiants, mais également les conditions atmosphériques à plus hautes altitudes ainsi que des régions éloignées qui se voient influencées, en raison des processus de transport atmosphériques - c'est-à-dire les vents - verticaux ou horizontaux. Par conséquent, 
un changement dans l'usage ou la couverture des sols, une modification dans la distribution des aires urbaines, agricoles ou forestières aura un effet sur la météorologie et le climat, à des échelles locales comme régionales et donc sur des distances allant du kilomètre à la dizaine, voire plusieurs centaines de kilomètres. Ces interactions entre surface et atmosphère ainsi que leurs mécanismes sous-jacents sont désormais bien connus du monde académique (Pielke et al., 2011). Ils ont pour la plupart été mis en équation et inclus dans des modèles de météorologie et de climatologie régionale (Pitman, 2003), ceux-là constituant des outils indispensables pour quantifier l'impact des changements d'usage et de gestion des sols sur les conditions atmosphériques. Ils permettent notamment d'évaluer, a posteriori ou par anticipation, l'impact de décisions aménagistes sur le climat.

\section{Mise en culture et impacts climatiques}

Une équipe de climatologues a pu s'intéresser aux effets d'un déplacement de cultures depuis les terres floridiennes plus septentrionales ou centrales vers des superficies, jadis marécageuses, localisées sur la partie méridionale de la péninsule (Pielke et al., 1999; Marshall et al., 2003; 2004a ; 2004b). Suite à une série de gels au cours de la décennie 1980, de nombreux producteurs d'agrumes avaient entrepris de déménager leurs productions en des régions plus méridionales de la péninsule floridienne, censées être moins sujettes au phénomène. Progressivement, ce sont de larges étendues qui furent consacrées sur ce territoire à la production de légumes d'hiver, de canne à sucre et d'agrumes (figure 3a-b- planche IV). Or, en janvier 1997, le monde agricole a dû faire face, sur cette partie méridionale de la péninsule floridienne, à un épisode de gel particulièrement long et vigoureux. Rien que pour les secteurs du légume d'hiver et de la canne à sucre, les dommages ont été estimés à près de 300 millions de dollars US sur les superficies les plus touchées par la vague de froid. En outre, près de 100000 travailleurs agricoles, souvent immigrés, se sont retrouvés sans emploi ou contraints à se déplacer pour en recouvrer un.

Cet événement météorologique a marqué les esprits, car si les épisodes de froid ne furent pas si rares dans le contexte floridien au cours du siècle dernier, ils touchaient davantage les parties septentrionales et centrales de la péninsule et non la partie méridionale. D'où l'hypothèse, émise par quelques scientifiques (Pielke et al., 1999), selon laquelle l'intensité de la vague de froid serait à chercher pour partie dans une modification de l'usage et de la couverture des sols. Une étude de modélisation a démontré que la conversion de ces superficies marécageuses en terres cultivées a altéré les flux d'énergie et d'eau échangés entre les surfaces et l'atmosphère, et par voie de conséquence les températures, notamment extrêmes, et la météorologie floridienne (Pielke et al., 1999; Marshall et al., 2004b). Différents épisodes thermiques extrêmes se sont vus intensifiés et prolongés dans le temps. Par exemple, dans le cas de la vague de froid de janvier 1997, les températures minimales auraient été jusqu'à $3{ }^{\circ} \mathrm{C}$ moins froides et la période de gel jusqu'à deux fois moins longue en certains endroits (figure 3c-d - planche IV) si la péninsule floridienne avait conservé l'utilisation et la couverture des sols antérieure de 1900 (Marshall et al., 2003; 2004a). Ce résultat est plutôt insolite dans le sens où les exploitants agricoles, en ayant entrepris de déplacer leurs cultures sur ces surfaces plus méridionales pour les tenir justement à l'abri du gel, ont paradoxalement intensifié et prolongé la vague de froid. Étant donné les coûts humains et économiques en jeu, on peut se demander si des études d'impact météorologique et climatique ne seraient pas à considérer en amont de changements d'importance en matière d'utilisation ou de gestion des sols.

\section{Expansion urbaine et impacts climatiques}

Un autre cas d'étude expose les effets attendus de l'expansion urbaine sur le climat, à des échelles locale et régionale, dans l'aire métropolitaine du Grand Phoenix (USA) (Georgescu et al., 2008; Georgescu et al., 2009a et b; Georgescu et al., 2012; Georgescu et al., 2013). L'Arizona Sun Corridor est l'une des conurbations croissant le plus vite dans le pays, les projections moyennes envisageant une population supérieure à 12 millions d'âmes en 2050 contre 5,5 millions d'habitants aujourd'hui. Sur cette base démographique, la Maricopa Association of Governments (MAG), à savoir l'agence de l'aire métropolitaine du Grand Phoenix chargée de mener 
des études en matière de planification spatiale et stratégique, a pu élaborer différents scenarii spatialisés de développement.

À partir des enveloppes urbaines extrapolées des différents scenarii d'expansion (figure 4 planche $\mathbf{V}$ ), ont été estimées les conséquences climatiques à attendre au titre des modifications induites dans les échanges énergétiques et hydrologiques par une artificialisation des sols. Le scénario d'expansion maximum de l'enveloppe urbaine entrainerait un réchauffement marqué des températures moyennes en période estivale comme hivernale, bien que cette hausse soit davantage circonscrite autour des aires nouvellement bâties dans le second cas. Ce réchauffement se surimposerait au réchauffement climatique attendu. Un scénario se basant sur cette même enveloppe urbaine élargie mais intégrant un recours massif aux toits à forte réflectivité, dit «froids ", induirait un réchauffement des températures bien plus limité en période estivale et davantage circonscrit géographiquement parlant (figure 5 - planche $\mathbf{V}$ ). Cette solution technique, permettant de limiter de manière marquée la hausse des températures en période estivale, aurait néanmoins un impact sur la pluviométrie avec une réduction plus importante des précipitations. Cet exemple expose à quel point il pourrait être pertinent de réaliser des études environnementales venant rendre compte des impacts météorologiques et climatiques susceptibles d'être produits par différents scénarii de planification.

\section{Plantation d'arbres et conséquences sur la qualité de l'air}

Le dernier cas d'étude sélectionné met en exergue les impacts sur la qualité de l'air d'une campagne de plantation d'arbres entreprise au cours des années 1980 et 1990 dans la région de Los Angeles pour réduire l'effet d'ICU et les coûts énergétiques liés à l'utilisation intensive de la climatisation en période estivale ou encore améliorer la qualité de l'air. Néanmoins, si la plantation d'arbres en milieu urbain peut offrir un effet rafraîchissant durant les épisodes de chaleur, le choix des arbres à planter s'avère fondamental (Corchnoy et al., 1992 ; Yang et al., 2005). Lorsque les oxydes d'azote (NOx), très présents en milieux urbains en raison des émissions liées aux activités ou à la circulation automobile, se voient mêlés à des COV, s'ensuit une formation d'ozone troposphérique, néfaste notamment pour la santé humaine. Dans le contexte de Los Angeles, le processus est d'autant plus vigoureux, étant donné le climat chaud et ensoleillé et une circulation atmosphérique influencée par la présence des montagnes proches de la côte pacifique. Certaines espèces d'arbres moyennement/fortement émettrices de bio-COV peuvent induire, dans des conditions particulières, des concentrations d'ozone proches de la valeur seuil fixée par l'Organisation Mondiale de la Santé (50 ppb) (Taha, 1996). Même si moins présents en ville, les bio-COV émis par les plantes sont plus réactifs que les COV d'origine anthropique et peuvent ainsi grandement affecter la qualité de l'air dans un milieu urbain déjà riche en NOx. Si une attention particulière doit être portée aux espèces d'arbres et d'arbustes à planter, c'est aussi l'environnement dans lequel celles-ci se développent qui est à considérer. Sur l'aire métropolitaine d'Atlanta, l'augmentation des températures moyennes estivales due au développement de l'ICU a induit une hausse des émissions de COV par les plantes, ce malgré une réduction de $20 \%$ des superficies de forêts dans la région considérée sur les 15 années étudiées. La pollution estivale relative à l'ozone troposphérique est restée identique sur cette période, alors qu'une politique de réduction de $50 \%$ des COV d'origine anthropique (solvants industriels, carburants) avait été mise en place pour la modérer (Cardelino et Chameides, 1990).

\section{VERS LE DÉVELOPPEMENT D'UNE ACTION CLIMATIQUE RÉGIONALE}

Les travaux académiques relatés précédemment, à travers la présentation de cas d'étude, permettent de prendre conscience de l'impact, souvent mésestimé par les aménageurs, que peuvent avoir les sociétés humaines sur leurs conditions de vie (températures, précipitations, vents, etc.) plus ou moins rapprochées en occupant l'espace et en usant des sols pour différentes finalités fonctionnelles (résidentiel, agricole, etc.). Plus indirectement, ils laissent également imaginer de nouvelles possibilités d'influer sur le climat, la météorologie ou encore la qualité de l'air au travers de démarches planificatrices se souciant de l'usage et de la gestion des sols ainsi que de leurs conséquences atmosphériques à une échelle 
régionale. Une telle orientation mérite d'autant plus d'attention dans un contexte de changement climatique global, notamment afin d'en modérer certains impacts à travers les territoires. Il en va du confort de vie et de la santé des populations, de la conservation des écosystèmes, ou encore de la viabilité de certaines activités économiques.

\section{En quête de nouveaux leviers d'action}

L'intérêt d'appréhender l'action climatique à une échelle régionale et donc de considérer de vastes périmètres comprenant espaces urbains, périurbains et ruraux, est avant tout à relier à la possibilité de nouveaux leviers d'action. Jusqu'à présent, les collaborations entre météorologues/climatologues et aménageurs visant à améliorer la prise en compte sur le terrain des conséquences atmosphériques se concentrent essentiellement sur des milieux largement bâtis et habités. Par conséquent, l'idée que les conditions atmosphériques d'un territoire, urbain ou rural, puissent en partie dépendre de l'utilisation des sols sur des espaces situés à plusieurs dizaines ou centaines de kilomètres, n'est pas encore pleinement appréhendée. Un tel tropisme pour les milieux urbains tend à limiter notamment les réflexions quant à l'influence des vastes couverts végétaux, qu'ils soient agricoles ou naturels, sur les conditions atmosphériques régionales. Par conséquent, se voit également entravée l'émergence opérationnelle de nouveaux leviers d'action climatique tirant parti de processus biophysiques et biogéochimiques à l'œuvre entre la surface et l'atmosphère. De récents développements scientifiques invitent pourtant à considérer davantage ces superficies agricoles et naturelles.

Alors qu'une attention relativement importante fut portée traditionnellement dans le monde académique aux impacts des changements d'usages des sols sur les conditions atmosphériques, se multiplient désormais des travaux pionniers s'intéressant à l'influence des modalités de gestion des sols et de leurs couverts. Les pratiques agricoles, et dans une moindre mesure forestières, sont notamment au cœur des réflexions, étant donné les vastes superficies concernées et in fine les conséquences sur la météorologie, le climat ou encore la qualité de l'air. Historiquement, c'est surtout l'influence de l'irrigation qui fut investiguée (Barnston et Schickedanz,
1984). Certaines études suggèrent que le recours à cette pratique, notamment sur les vastes superficies cultivées des Grandes Plaines américaines, pourrait avoir entraîné une augmentation de la couverture nuageuse et des précipitations en période estivale (DeAngelis et al., 2010; Huber et al., 2014). L'effet pourrait avoir porté jusqu'à $90 \mathrm{~km}$ de distance sous le vent (Moore et Rojstaczer, 2002). D'autres travaux ont pu estimer, dans le contexte californien, l'impact de l'irrigation sur les températures et établir un effet rafraîchissant sur les températures moyennes diurnes (Bonfils et Lobell, 2007). Par ailleurs, des auteurs ont pu s'intéresser aux conséquences météorologiques que pourrait avoir le recours à une agriculture sans labour sur le continent européen, identifiant alors un effet rafraîchissant sur les températures maximales en été, juste après la récolte (Davin et al., 2014). Ce sont également des pratiques forestières telles que l'éclaircissement des massifs ou le passage de boisements feuillus en conifères qui ont pu être investigués, afin d'en estimer les impacts climatiques. (Luyssaert et al., 2014; Naudts et al., 2016). Il se devine ainsi, à travers ces aspects de gestion des sols et de leurs couverts, un potentiel à ne pas négliger en termes de leviers d'action pour agir sur les conditions atmosphériques. Ainsi que l'ont montré Naudts et al. (2016) dans un article traitant de l'impact de la gestion des forêts sur le climat en Europe, les modalités de gestion sont susceptibles d'avoir un impact sur le climat potentiellement aussi important que ceux induits par un changement d'usage des sols.

L'échange des connaissances entre climatologues/météorologues et aménageurs nous semble aujourd'hui trop limité, si l'on fait exception des collaborations engagées sur les milieux urbains. Nous proposons ainsi d'établir un dialogue fertile et constructif. Ce rapprochement viserait d'une part l'identification de nouveaux leviers d'action et par conséquent de mesures types à mettre en œuvre sur le terrain (tableau 1). Il permettrait d'autre part le développement d'outils d'anticipation des impacts de différents scénarios d'aménagement et de gestion des sols sur les conditions atmosphériques. Il serait par exemple envisageable d'évaluer les conséquences de ces scénarios sur a) les cycles saisonniers de la température, des précipitations et du vent (et donc le confort météorologique), b) la pollution de l'air à diverses périodes de l'année (et donc la santé 
du vivant), c) l'amplitude d'un événement extrême (et donc non seulement le confort mais également la santé). Cet ensemble de diagnostics pourrait s'opérer non seulement sur l'ensemble du territoire, mais également sur un maillage relativement fin $(\sim 1 \mathrm{~km})$ et offrant de quantifier les conséquences des changements envisagés sur les diverses composantes du territoire (sur les zones agricoles comme urbaines), sur les productions (agricoles, fourragères, forestières) et la ressource en eau.

\section{Défis en matières climatologique et planificatrice}

La collaboration, qu'il nous semble indispensable de mettre en œuvre, implique cependant que climatologues et aménageurs soient respectivement en mesure de relever certains défis.

Il existe tout d'abord une problématique d'outillage. Les politiques climatiques établies à destination des territoires, que ce soit dans une perspective globale d'atténuation de l'effet de serre ou dans une optique plus locale de lutte contre l'ICU, parviennent à appréhender la problématique de manière relativement indirecte, en préconisant l'adoption de mesures génériques censées fonctionner dans la plupart des contextes. Il peut ainsi être recommandé de suivre certains principes d'aménagement en vue de réduire les émissions de gaz à effet de serre ou de limiter l'élévation des températures dans les milieux bâtis. Autrement dit, ces politiques s'avèrent largement efficientes dans le sens où elles permettent l'économie d'une compétence climatique approfondie chez ceux qui les mettent en œuvre au cœur des territoires. En revanche, le développement d'une action climatique d'échelle intermédiaire, visant à tirer profit des connaissances en matière d'interaction surface-atmosphère exposés précédemment, peut difficilement se concevoir sans recours aux modèles météorologiques ou climatiques régionaux, puisqu'une même préconisation en matière d'usage ou de gestion des sols peut avoir des conséquences très variables, voire inverses, selon les contextes.

Or, les modèles dont disposent les climatologues n'ont encore jamais réellement été éprouvés aux échelles spatiales intermédiaires sur lesquelles l'action pourrait se voir esquissée. Ils sont le plus souvent utilisés pour réaliser de la prévision météorologique ou sinon pour étudier les évolutions climatiques sur un pays ou un continent, et ce à un maillage (une résolution horizontale) le plus souvent supérieur à $15 \mathrm{~km} \times 15 \mathrm{~km}$. Pour étudier l'évolution de la météorologie au-dessus de tissus urbains, ces mêmes modèles se basent sur un maillage de l'ordre du kilomètre de côté. C'est pourtant bien une échelle intermédiaire qu'il nous semble important d'explorer désormais et pour laquelle des développements en modélisation seront peut-être à faire. Il est certain que la description des surfaces et de leurs usages dans ces modèles reste aujourd'hui trop sim-

\begin{tabular}{|l|l|l|l|}
\hline Échelle spatiale & \multicolumn{1}{|c|}{ Local } & \multicolumn{1}{|c|}{ Régional } & \multicolumn{1}{c|}{ Global } \\
\hline Mesures types & Végétaliser les villes & $\begin{array}{l}\text { Orienter l'usage (dans l'optique } \\
\text { d'une bonne répartition et propor- } \\
\text { tion entre espaces urbains, fores- } \\
\text { tiers, agricoles) et la gestion des } \\
\text { sols (pratiques agricoles, gestions } \\
\text { forestières, etc.) à des échelles } \\
\text { métropolitaines ou régionales } \\
\text { élargies }\end{array}$ & $\begin{array}{l}\text { Organiser spatialement la ville et } \\
\text { les réseaux de transport de manière } \\
\text { à limiter les émissions carbonées } \\
\text { relatives aux mobilités, notamment } \\
\text { individuelles }\end{array}$ \\
\hline Effets attendus & $\begin{array}{l}\text { Rafraîchir les milieux urbains, } \\
\text { en vue notamment de modérer } \\
\text { l'augmentation des températures } \\
\text { redoutée dans un contexte de } \\
\text { réchauffement climatique mondial }\end{array}$ & $\begin{array}{l}\text { Limiter les pollutions atmosphé- } \\
\text { riques néfastes à la santé humaine } \\
\text { et végétale sur le territoire } \\
\text { Améliorer le confort hydrique } \\
\text { et thermique sur les superficies } \\
\text { urbaines et rurales, aujourd'hui } \\
\text { comme dans un contexte clima- } \\
\text { tique plus chaud }\end{array}$ & $\begin{array}{l}\text { Diminuer la concentration de gaz } \\
\text { à effet de serre dans l'atmosphère } \\
\text { afin de limiter le réchauffement } \\
\text { climatique mondial }\end{array}$ \\
\hline
\end{tabular}

Tableau 1 : Mesures types envisageables et leurs effets attendus sur le climat, la météorologie ou la qualité de l'air à différentes échelles spatiales

Example measures and expected effects on climate, weather and air quality at multiple spatial scales 
plifiée, même si elle n'empêche pas le lancement de premières études de cas. Les climatologues doivent donc d'ores et déjà s'atteler à inclure une représentation des surfaces qui soit plus proche de celles sur laquelle travaillent les aménageurs et planificateurs.

Étant donné l'influence que peuvent avoir l'usage des sols ainsi que la gestion de leurs couverts dans les processus environnementaux précédemment décrits, les planificateurs pourraient gagner un rôle élargi en matière d'action sur le climat et la qualité de l'air. Néanmoins, cela suppose de leur part une capacité à intégrer dans leurs réflexions et pratiques de nouvelles considérations relativement complexes. C'est aussi le contour du champ de la planification qui pose question, la discipline étant historiquement tournée vers l'occupation des sols. Les codes de l'urbanisme nationaux permettent de spécifier au travers du zonage un usage spécifique et de déterminer ainsi des aires à caractère résidentiel, industriel, commercial, agricole ou encore naturel. En revanche, les planificateurs n'ont généralement pas compétence à définir ou orienter des pratiques de gestion des sols, notamment dans le registre agricole ou forestier. Or, cette problématique climatique invite à considérer dans les réflexions planificatrices des aspects ayant également trait à la gestion des sols. Certains instruments tels que les mesures agroenvironnementales et climatiques peuvent constituer des leviers pour orienter la gestion des sols, mais l'inscription de tels dispositifs incitatifs dans la pratique planificatrice reste à effectuer. Certains auteurs ont pu déplorer le défaut d'une planification intégrée permettant de localiser plus finement les mesures agroenvironnementales et climatiques dans le territoire pour que celles-ci portent réellement leurs fruits (Haaren et Bathke, 2008; Prager et al., 2012). Des méthodologies ont été développées en ce sens mais restent pour lors peu usitées par les praticiens de la planification.

Un autre défi concerne la territorialisation de cette action climatique d'échelle intermédiaire, ou autrement dit son inscription dans le système de planification et dans l'architecture administrative. Pour bien des champs environnementaux, il est possible d'identifier une biorégion (Giraut, 2011; Brunckhorst, 2013) ou autrement dit un périmètre géographiquement pertinent au travers duquel sera appréhendé l'écosystème ou la ressource en question. Il existe par exemple une certaine logique envi- ronnementale à traiter des enjeux relatifs à l'eau à l'échelle des bassins-versants. Or, pour cette question climatique intermédiaire, il est difficile d'établir un périmètre géographique pertinent, sachant que les processus considérés peuvent courir sur des distances variables et qu'une même occupation des sols peut avoir des conséquences distinctes selon les orientations. Dans le contexte français, il est en revanche prévu d'appréhender les aspects climatiques à l'échelle de la région administrative en l'occurence à travers l'adoption prochaine de schémas régionaux d'aménagement, de développement durable et d'égalité des territoires (SRADDET) dans lesquels sont censés se fondre les schémas régionaux climat-air-énergie (SRCAE) adoptés jusqu’à lors. Ces derniers avaient été pensés lors de leur instauration comme un rouage essentiel pour la mise en œuvre territoriale des stratégies d'atténuation et d'adaptation au changement climatique global. Le SRADDET - dont les objectifs et règles générales sont censés s'imposer aux documents d'urbanisme respectivement dans un rapport de prise en compte et de compatibilité - pourrait ainsi constituer le cadre indiqué pour développer cette action climatique d'échelle intermédiaire.

\section{Conclusion}

Cette note vise à convaincre aménageurs et climatologues de l'intérêt d'un travail en commun. Il existe certes des exemples de collaboration fructueuse, mais de telles démarches ont pour lors essentiellement porté sur des milieux urbains et des périmètres relativement restreints. Les climatologues ont pourtant pu démontrer l'influence que peuvent avoir les sols et leurs couverts, notamment végétaux, sur les conditions atmosphériques proches ou plus distantes. Certains de ces travaux ont d'ailleurs été restitués ici-même à travers la présentation de cas d'étude puisés dans la littérature académique. Des modifications dans l'usage des sols ou dans la gestion de leurs couverts peuvent ainsi influer les températures et précipitations sur de vastes territoires. De telles considérations s'avèrent particulièrement précieuses dans un contexte de changement climatique global. Il est en effet envisageable de pouvoir modérer dans les territoires certaines perturbations chroniques ou les effets d'événements extrêmes tels que des canicules, cela notamment dans l'idée 
de limiter les répercussions négatives en termes de confort et de santé publique, mais aussi de productivité agricole par exemple. D'autre part, ce sont également des aspects ayant trait à la qualité de l'air, certaines pollutions affectant tout autant les populations que les cultures, qui sont en jeu. Certaines espèces végétales, et même des pratiques agricoles telles que la fertilisation, induisent des émissions qui peuvent être in fine nocives. Pour toutes ces raisons, nous suggérons d'étendre le champ de l'action climatique considéré en aménagement, notamment dans le cadre de réflexions planificatrices appréhendant les sols à une échelle élargie, afin de pouvoir tirer pleinement profit des connaissances en matière d'interactions surfaces-atmosphère et ainsi mobiliser de nouveaux leviers.

\section{Remerciements}

Ce travail a été réalisé dans le cadre du Laboratoire d'excellence BASC (Biodiversité, Agrosystèmes, Société, Climat), financé par l'Agence de Recherche nationale française (ANR) à l'issue de l'appel à projets sur «les Investissements d'Avenir » (LabEx BASC; ANR-11-LABX-0034). Deux des auteurs ont été financés par BASC. L'ensemble des auteurs remercient le LabEx BASC qui a permis de réunir sur un même projet chercheurs en sciences sociales et climatologie.

\section{Bibliographie}

BACH W., 1972. Urban climate, air pollution and planning, in Detwyler T.R., Marcus M.G. (eds), Urbanization and Environment: The Physical Geography of the City, Duxbury Press, Belmont (CA), p. 69-96.

Barnston A.G., Schickedanz P.T., 1984. The effect of irrigation on warm season precipitation in the southern Great Plains, Journal of Applied Meteorology, 23 (6), p. 865-888.

Baumüller J., Reuter U., Hoffmann U., Esswein H., 2008. Klimaatlas Region Stuttgart, Verband Region Stuttgart, Stuttgart, Schriftenreihe Verband Region Stuttgart, 26, 50 p.

Bonfils C., Lobell D., 2007. Empirical evidence for a recent slowdown in irrigation-induced cooling, Proceedings of the National Academy of Sciences, 104 (34), p. 13583-13587.

Brunckhorst D.J., 2013. Bioregional Planning: resource management beyond the new millennium, Londres/New York, Routledge, 200 p.
Bulkeley H., 2006. A changing climate for spatial planning?, Planning Theory and Practice, 7 (2), p. 203-214.

Cardelino C.A., Chameides W.L., 1990. Natural hydrocarbons, urbanization, and urban ozone, Journal of Geophysical Research, 95, p. 13971-13979.

Chameides W.L., Lindsay R.W., Richardsen J., Kiang C.S., 1988. The role of biogenic hydrocarbons in urban photochemical smog: Atlanta as a case study, Science, 241 (4872), p. 1473-1475.

Corchnoy S.B., Arey, J., Atkinson R., 1992. Hydrocarbon emissions from twelve urban shade trees of the Los Angeles, California, air basin, Atmospheric Environment, 26B, p. 339-348.

Cordeau E., 2014. La vulnérabilité de la ville à la chaleur par l'approche zones climatiques locales, Note rapide Environnement, 661, 6 p.

Davin E.L., Seneviratne S.I., Ciais P., Olioso A., Wang T., 2014. Preferential cooling of hot extremes from cropland albedo management, Proceedings of the National Academy of Sciences, 11 (27), p. 9757-9761.

Davoudi S., Crawford J., Mehmood A., 2009. Planning for Climate Change: Strategies for Mitigation and Adaptation for Spatial Planners, London, EARThSCAN, 344 p.

DeAngelis A., Dominguez F., Fan Y., Robock A., Kustu M.D., Robinson D., 2010. Evidence of enhanced precipitation due to irrigation over the Great Plains of the United States, Journal of Geophysical Research D: Atmospheres, 115 (15), p. D15115.

Food and Agriculture Organization (FAO), United Nations Development Programme (UNDP), United Nations Environment Programme (UNEP). United Nations Programme on Reducing Emissions from Deforestation and Forest Degradation (REDD Programme) [http:// www.un-redd.org/].

Georgescu M., Miguez-Macho G., Steyaert L.T., Weaver C.P., 2008. Sensitivity of summer climate to anthropogenic land-cover change over the Greater Phoenix, AZ, region, Journal of Arid Environments, 72, p. 1358-1373.

Georgescu M., Miguez-Macho G., Steyaert L.T., Weaver C.P., 2009a. Climatic effects of 30 years of landscape change over the Greater Phoenix, Arizona, region: 1. Surface energy budget changes, Journal of Geophysical Research, 114, D05110, doi:10.1029/2008JD010745.

Georgescu M., Miguez-Macho G., Steyaert L.T., Weaver C.P., 2009b. Climatic effects of 30 years of landscape change over the Greater Phoenix, Arizona, region: 2. Dynamical and thermodynamical response, Journal of Geophysical Research, 114, D05111, doi:10.1029/2008JD010762.

Georgescu M., Mahalov A., Moustaoui M., 2012. Seasonal hydroclimatic impacts of Sun Corridor expansion, Environmental Research Letters, 7 (3), p. 34026-34035.

Georgescu M., Moustaoui M., Mahalov A., Dudhia J., 2013. Summer-time climate impacts of projected megapolitan expansion in Arizona, Nature Climate Change, 3, p. 37-41.

Giraut F., 2011 . Bioregionalization and territorial complexity in the global South, Procedia Social and Behavioral Sciences, 14, p. 49-52. 
HaAren C., Bathke M., 2008. Integrated landscape planning and remuneration of agri-environmental services. Results of a case study in the Fuhrberg region of Germany, Journal of Environmental Management, 89, p. 209-221.

Heald C.L., Spracklen D.V., 2015. Land Use Change Impacts on Air Quality and Climate, Chemical Reviews, 115 , p. 4476-4496.

Hebbert M., Webв B., 2012. Towards a Liveable Urban Climate: Lessons from Stuttgart, in Christopher G. (ed.), Liveable Cities: Urbanising World: ISOCARP Review 07, London, Routledge, p. 132-150.

Hebbert M., Jankovic V., 2013. Cities and Climate Change: The Precedents and Why They Matter, Urban Studies, 50 (7), p. 1332-1347.

Hebbert M., 2014. Climatology for City Planning in Historical Perspective, Urban Climate, 10, p. 204-215.

Huber D.B., Мechem D.B., Brunsell N.A., 2014. The Effects of Great Plains Irrigation on the Surface Energy Balance, Regional Circulation, and Precipitation, Climate, 2 (2), p. 103-128.

Luyssaert S., Jammet M.M., Stoy P.C., Estel S., Pongratz J., Ceschia E., Churkina G., Don A., Erb K., Ferlicoq M., Gielen B., Gruenwald T., Houghton R.A., Klumpp K., Knohl A., Kolb T., Kuemmerle T., Laurila T., Lohila A., Loustau D., McGrath M.J., Meyfroidt P., Moors E.J., Naudts K., Novick K., Otto J., Pilegaard K., Pio C.A., Rambal S., Rebmann C., Ryder J., Suyker A.E., Varlagin A., Wattenbach M., Dolman A.J., 2014. Land management and land-cover change have impacts of similar magnitude on surface temperature, Nature Climate Change, 4, p. 389-393.

Mahmood R., Pielke Sr. R.A., Hubbard K.G., Niyogi D., Dirmeyer P.A., McAlpine C., Carleton A.M., Hale R., Gameda S., Beltrán-Przekurat A., Baker B., Mc Nider R., Legates D.R., Shepherd M., Du J., Blanken P.D., Frauenfeld O.W., Nair U.S., Fall S., 2014. Land cover changes and their biogeophysical effects on climate, International Journal of Climatology, 23, p. 929-953.

Marshall C.H., Pielke Sr. R.A., Steyaert L.T., 2003. Crop freezes and land-use change in Florida, Nature, 426, p. 29-30.

Marshall C.H., Pielke Sr. R.A., Steyaert L.T., 2004a. Has the Conversion of Natural Wetlands to Agricultural Land Increased the Incidence, and Severity of Damaging Freezes in South Florida?, Monthly Weather Review, 132, p. 22432258.

Marshall C.H., Pielke Sr. R.A., Steyaert L.T., Willard D.A., 2004b. The Impact of Anthropogenic Land-Cover Change on the Florida Peninsula Sea Breezes and Warm Season Sensible Weather, Monthly Weather Review, 132, p. $28-52$.

Masson V., Lion Y., Peter A., Pigeon G., Buyck J., Brun E., 2013. "Grand Paris": Regional landscape change to adapt city to climate warming, Climatic Change, 117 (4), p. 769 782.

Ministère de L'Agriculture, de L'Agroalimentaire et dE LA ForÊT. Programme 4 pour 1000 [http://4p 1000.org/].
Moore N., Rojstaczer S., 2002. Irrigation's influence on precipitation: Texas High Plains, USA, Geophysical Research Letters, 29(16), doi:10.1029/2002GL014940.

Naudts K., Chen Y., McGrath M.J., Ryder J., Valade A., Otтo J., Luyssaert S., 2016. Europe's forest management did not mitigate climate warming, Science, 351 (6273), p. 597-600.

Ng E, Ren C., 2015. The Urban Climatic Map: A Methodology for Sustainable Urban Planning, London/New York, Routledge, $528 \mathrm{p}$.

Nowak D.J., Civerolo K.L., Rao S.T., Sistla G., Luley C.J., Crane D.E., 2000. A modelling study of the impact of urban trees on ozone, Atmospheric Environment, 34, p. 1601-1613.

Pielke R.A., Walko R.L., Steyaert L.T., Vidale P.L., Liston G.E., Lyons W.A., Chase T.N., 1999. The influence of anthropogenic landscape changes on weather in south Florida, Monthly Weather Review, 127, p. 1663-1673.

Pielke R.A., Pitman A., Niyogi D., Mahmood R., McAlpine C., Hossain F., Klein Goldewijk K., Nair U., Betts R., Fall S., Reichstein M., Kabat P., de Noblet-Ducoudré N., 2011 . Land use/land cover changes and climate: modeling analysis and observational evidence, WIREs Climate Change, 2, p. 828-850.

Pitman A. J., 2003. The evolution of, and revolution in, land surface schemes designed for climate models, International Journal of Climatology, 23 (5), p. 479-510.

Pitman A., de Noblet-Ducoudré N., 2011 . Human effects on climate through land-use induced land cover change, in Henderson-Sellers A., McGuffie K. (eds.) The Future of the World's Climate, Amsterdam, Elsevier, p. 77-95.

Prager K., Reed M., Scott A., 2012. Encouraging collaboration for the provision of ecosystem services at a landscape scale. Rethinking agri-environmental payments, Land Use Policy, 29, p. 244-249.

RADDATz R.L., 2007. Evidence for the influence of agriculture on weather and climate through the transformation and management of vegetation: Illustrated by examples from the Canadian Prairies, Agricultural and Forest Meteorology, 142, p. 186-202.

Ren C., NG E., Katzchner L., 2010. Urban climatic map studies: a review, International Journal of Climatology, 31 (15), p. 2213-2233.

Seneviratne S.I., Corti T., Davin E.L., Hirschi M., Jaeger E.B., Lehner, Orlowsky B., Teuling A.J., 2010. Investigating soil moisture-climate interactions in a changing climate: A review, Earth-Science Reviews, 99 (3-4), p. 125-161.

Sillman S., 1999. The relation between ozone, NOx and hydrocarbons in urban and rural environments, Atmospheric Environment, 33, p. 1821-1845.

Stewart I.D., Oke T.R., 2012. Local Climate Zones for urban temperature studies, Bulletin of the American Meteorological Society, 93, p. 1879-1900.

ТАна H., 1996. Modelling impacts of increased urban vegetation on ozone air quality in the South Coast Air Basin, Atmospheric Environment, 30, p. 3423-3430. 
Upmanis H., Eliasson I., Linqvist S., 1998. The influence of green areas on nocturnal temperatures in a high latitude city (Göteborg, Sweden), International Journal of Climatology, 18 (6), p. 681-700.

Wilson E., Piper J., 2006. Spatial Planning and Climate Change, Londres/New York, Routledge, 480 p.
Yang J., Mcbride J., Zhou J., Sun Z., 2005. The urban forest in Beijing and its role in air pollution reduction, Urban Forestry et Urban Greening, 3, p. 65-78. 